\title{
Cardiac output estimation by multi-beat analysis of the radial arterial blood pressure waveform versus intermittent pulmonary artery thermodilution: a method comparison study in patients treated in the intensive care unit after off-pump coronary artery bypass surgery
}

\author{
Gillis Greiwe $^{1} \cdot$ Valerie Peters $^{1} \cdot$ Alexander Hapfelmeier $^{2} \cdot$ Stefano Romagnoli $^{3,4} \cdot$ Mathias Kubik $^{5,6} \cdot$ Bernd Saugel $^{1,7}$ (D)
}

Received: 18 April 2019 / Accepted: 5 August 2019 / Published online: 27 August 2019

(C) Springer Nature B.V. 2019

\begin{abstract}
Cardiac output (CO) is a key hemodynamic variable that can be minimally invasively estimated by pulse wave analysis. Multi-beat analysis is a novel pulse wave analysis method. In this prospective observational clinical method comparison study, we compared $\mathrm{CO}$ estimations by multi-beat analysis with $\mathrm{CO}$ measured by intermittent pulmonary artery thermodilution (PATD) in adult patients treated in the intensive care unit (ICU) after off-pump coronary artery bypass surgery (OPCAB). We included patients after planned admission to the ICU after elective OPCAB who were monitored with a radial arterial catheter and a pulmonary artery catheter. At seven time points, we determined CO using intermittent PATD (PATD-CO; reference method) and simultaneously recorded the radial arterial blood pressure waveform that we later used to estimate CO using multi-beat analysis (MBA-CO; test method) with the Argos monitor (Retia Medical; Valhalla, NY, USA). Blood pressure waveforms impaired by inappropriate damping properties or artifacts were excluded. We compared PATD-CO and MBA-CO using Bland-Altman analysis accounting for repeated measurements, the percentage error, and the concordance rate derived from four-quadrant plot analysis (15\% exclusion zone). We analyzed 167 CO values of 31 patients. Mean PATD$\mathrm{CO}$ was $5.30 \pm 1.22 \mathrm{~L} / \mathrm{min}$ and mean MBA-CO was $5.55 \pm 1.82 \mathrm{~L} / \mathrm{min}$. The mean of the differences between PATD-CO and MBA-CO was $0.08 \pm 1.10 \mathrm{~L} / \mathrm{min}(95 \%$ limits of agreement: $-2.13 \mathrm{~L} / \mathrm{min}$ to $+2.29 \mathrm{~L} / \mathrm{min})$. The percentage error was $40.7 \%$. The four-quadrant plot-derived concordance rate was $88 \%$. CO estimation by multi-beat analysis of the radial arterial blood pressure waveform (Argos monitor) shows reasonable agreement compared with CO measured by intermittent PATD in adult patients treated in the ICU after OPCAB.
\end{abstract}

Keywords Pulmonary artery catheter $\cdot$ Swan-Ganz catheter $\cdot$ Hemodynamic monitoring $\cdot$ Pulse wave analysis $\cdot$ Pulse contour analysis $\cdot$ Cardiovascular dynamics $\cdot$ Anesthesia

Bernd Saugel

bernd.saugel@gmx.de; b.saugel@uke.de

1 Department of Anesthesiology, Center of Anesthesiology and Intensive Care Medicine, University Medical Center Hamburg-Eppendorf, Martinistrasse 52, 20246 Hamburg, Germany

2 Institute of Medical Informatics, Statistics and Epidemiology, Klinikum rechts der Isar, Technische Universität München, Munich, Germany

3 Department of Health Science, Section of Anesthesia and Critical Care, University of Florence, Florence, Italy
4 Department of Anesthesia and Critical Care, AOU Careggi, Florence, Italy

5 Department of Cardiovascular Surgery, University Heart Center Hamburg, Hamburg, Germany

6 Department of Intensive Care Medicine, Center of Anesthesiology and Intensive Care Medicine, University Medical Center Hamburg-Eppendorf, Hamburg, Germany

7 Outcomes Research Consortium, Cleveland, OH, USA 


\section{Introduction}

Cardiac output $(\mathrm{CO})$ - as a main determinant of oxygen delivery-is a key hemodynamic variable to guide therapy in critically ill patients with circulatory shock [1]. Invasive indicator dilution methods such as pulmonary artery thermodilution (PATD) [2] and transpulmonary thermodilution [3] remain the clinical reference methods to assess $\mathrm{CO}$ and novel minimally-invasive hemodynamic monitoring tools should be validated against these invasive reference methods [4-6]. Invasive uncalibrated pulse wave analysis requiring only an arterial catheter is increasingly used as a minimally invasive method for stroke volume-and thus $\mathrm{CO}$ - estimation in surgical and critically ill patients [5-7]. The term pulse wave analysis comprises several markedly different approaches and algorithms to estimate stroke volume by analyzing the arterial blood pressure waveform [8, 9]. As a main advantage, pulse wave analysis enables $\mathrm{CO}$ to be estimated continuously. It is therefore recommended for real-time $\mathrm{CO}$ estimation during functional tests of fluid responsiveness such as the passive leg raising [10] or fluid challenge test [11].

Multi-beat analysis is a novel method to estimate stroke volume by mathematically analyzing the radial blood pressure waveform over time scales greater than a single cardiac cycle without external calibration. This enables beatto-beat waveform variations to be considered and effects of pulse wave reflections to be minimized; eventually, by analyzing the waveform over several cardiac cycles, the multi-beat analysis method aims to identify the pure exponential pressure decay of the arterial blood pressure waveform without confounding wave reflections in the periphery of the arterial vessel tree and estimates stroke volume using a proprietary formula and biometric data [12, 13]. Based on experimental [13] and retrospective [12, 14, 15] pilot studies Retia Medical (Valhalla, NY, USA) developed a commercially available bedside $\mathrm{CO}$ monitor using multi-beat analysis - the Argos monitor-that was recently cleared by the US Food and Drug Administration (FDA). In this clinical method comparison study, we compared $\mathrm{CO}$ estimations by multi-beat analysis of the radial arterial blood pressure waveform (Argos monitor) to $\mathrm{CO}$ measured by intermittent PATD in adult patients treated in the intensive care unit (ICU) after off-pump coronary artery bypass surgery (OPCAB).

\section{Methods}

\subsection{Study design and protocol}

This was a CO method comparison study performed in the Center of Anesthesiology and Intensive Care Medicine at the University Medical Center Hamburg-Eppendorf (Hamburg, Germany) between August 2017 and February 2018. The ethics committee approved this study (Ethikkommission der Ärztekammer Hamburg, Hamburg, Germany) and participants gave written informed consent prior to enrollment. Adult patients (age $\geq 18$ years) who were scheduled for elective OPCAB with planned postoperative ICU admission and in whom hemodynamic monitoring with a radial artery catheter and a pulmonary artery catheter (PAC) was planned for clinical reasons not related to the study were eligible for inclusion in the study. Patients could not be included if they could not give or refused to give informed consent or had severe heart valve insufficiency or arrhythmias. Patients were excluded if the clinical situation did not allow for conducting study measurements (e.g. agitated patient, transfer to the OR for redo surgery, removal of the arterial line or the PAC).

After the surgical procedure, patients were transferred to the ICU and standard monitoring was established. Correct positioning of the PAC was verified radiographically and by continuous pulmonary artery pressure assessment. After admission to the ICU, we measured CO using PATD (PATD-CO; reference method) at seven time points (with approximately $30 \mathrm{~min}$ in between sets) and simultaneously recorded the radial blood pressure waveform for later offline estimation of $\mathrm{CO}$ using multi-beat analysis (MBA-CO; test method; Argos monitor, Retia Medical, Valhalla, NY, USA). No study related intervention was performed. Patients were treated according to routine care in the operating room and in the ICU.

\subsection{Study measurements}

At each of the seven time points, we performed and averaged five single PATD measurements, each performed by injecting a $10 \mathrm{~mL}$ bolus of ice-cold saline solution. We used a 7 French thermodilution pulmonary artery catheter (PAC) (Arrow; Teleflex Medical Europe Ltd; Co Westmeath, Ireland). Single PATD boluses were randomly injected over the respiratory cycle. For the time span of each set of PATD measurements we extracted the blood pressure signal sampled at $100 \mathrm{~Hz}$ from the ICU monitoring network via a data grabbing software (Dräger Data Grabber; Dräger; Lübeck, Germany). Waveform segments starting $20 \mathrm{~s}$ before the first and stopping $20 \mathrm{~s}$ after the last 
PATD measurement were included in the analysis. These blood pressure waveform segments were fed into the Argos monitor for off-line analysis via a $3.5 \mathrm{~mm}$ stereo cable, using an MP150 digital-to-analog converter (BIOPAC System; Goleta, CA, USA). Required patient characteristics (gender, age, height, body weight) were entered into the Argos monitor. We used the Argos CO monitor in the 20-s mode, i.e., the monitor estimates and displays $\mathrm{CO}$ every $5 \mathrm{~s}$ using multi-beat analysis of the arterial blood pressure waveform over $20 \mathrm{~s}$. MBA-CO values estimated during the time span of the corresponding PATD-CO measurements were averaged.

Before the final analysis, three researchers independently visually inspected the recorded arterial blood pressure waveforms for inappropriate damping properties (underdamping/ resonance artifacts or overdamping) and artifacts (flushing of the arterial line or motion artifacts). Waveforms impaired by inappropriate damping properties (missed during routine clinical care despite regularly performed fast-flush tests [16]) or artifacts were excluded from further analysis.

\subsection{Statistical analysis}

We calculated the mean \pm standard deviation (SD) to describe continuous patient data and absolute and relative frequencies to describe qualitative patient characteristics. We calculated the mean $\mathrm{CO}$ values \pm SD for PATD-CO and MBA-CO. The correlation between PATD-CO and MBA$\mathrm{CO}$ was computed within subjects taking repeated observations into account and removing the between subject variability (as required for method comparison studies) [17]. To assess the agreement between PATD-CO and MBA-CO we performed Bland-Altman analysis accounting for multiple observations per patient [18] and calculated the mean and SD of the differences, the $95 \%$ limits of agreement (=mean difference $\pm 1.96 \times \mathrm{SD}$ of the difference), and the percentage error [19]. To assess the trending ability of MBA-CO compared with PATD-CO we calculated the concordance rate based on four-quadrant plot analysis showing relative $\mathrm{CO}$ changes using a central exclusion zone of $15 \%$ [20, 21]. For statistical analyses we used $\mathrm{R}$ version 3.5.0 (The R Foundation for Statistical Computing, Vienna, Austria).

\section{Results}

A total of 284 paired PATD-CO and MBA-CO measurements in 42 patients were recorded. Two patients had to be excluded because of malposition of the PAC and one patient was excluded because of excess motion artifacts of the blood pressure waveform. 106 segments were excluded because of inappropriate damping properties or artifacts
Table 1 Baseline patient characteristics

\begin{tabular}{ll}
\hline $\begin{array}{l}\text { Number of } \\
\text { included patients } \\
(\mathrm{n})\end{array}$ & 31 \\
Age (years) & $71 \pm 12$ \\
Sex, female n $(\%)$ & $6(19)$ \\
Height $(\mathrm{m})$ & $1.74 \pm 0.08$ \\
Weight $(\mathrm{kg})$ & $87 \pm 19$ \\
BMI $\left(\mathrm{kg} / \mathrm{m}^{2}\right)$ & $28.8 \pm 5.8$ \\
\hline
\end{tabular}

Data are displayed as absolute and relative frequencies or mean and standard deviation

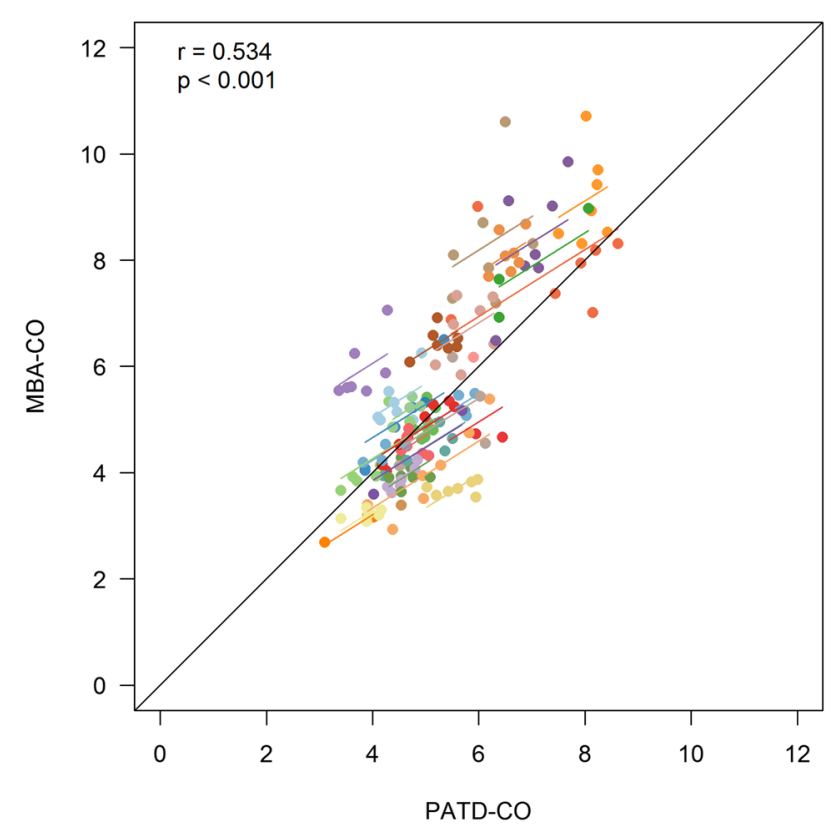

Fig. 1 Scatter plot illustrating the relation of cardiac output estimations obtained with multi-beat analysis (MBA-CO; test method) and cardiac output measurements with pulmonary artery thermodilution (PATD-CO; reference method). The within-subject correlation coefficient $(r)$ is presented along a respective illustration of the intra-individual linear trend. Patients are distinguished by color

of the blood pressure waveform. We included 167 paired $\mathrm{CO}$ values of 31 patients in the final analysis.

Basic patient characteristics are shown in Table 1. The relation and within-subject correlation of PATD-CO and MBA-CO is shown in Fig. 1. Mean PATD-CO and MBA-CO were $5.30 \pm 1.22 \mathrm{~L} / \mathrm{min}$ and $5.55 \pm 1.82 \mathrm{~L} / \mathrm{min}$, respectively. Bland-Altman analysis revealed a mean of the differences between PATD-CO and MBA-CO of $0.08 \pm 1.10 \mathrm{~L} / \mathrm{min}(95 \%$ limits of agreement: $-2.13 \mathrm{~L} /$ $\min$ to $+2.29 \mathrm{~L} / \mathrm{min}$ ) (Fig. 2). The percentage error was $40.7 \%$. Four-quadrant plot analysis is shown in Fig. 3. The concordance between changes in PATD-CO and MBA-CO was $88 \%$. 


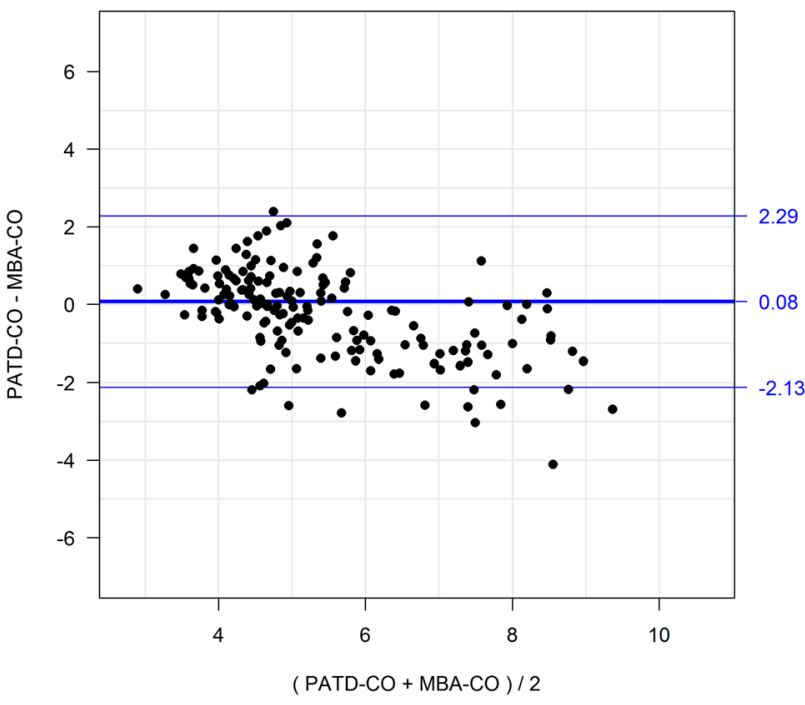

Fig. 2 Bland-Altman plot illustrating mean of the differences (bold horizontal line) and 95\% limits of agreement (upper and lower thin horizontal lines) between the reference method (PATD-CO) and the test method (MBA-CO)

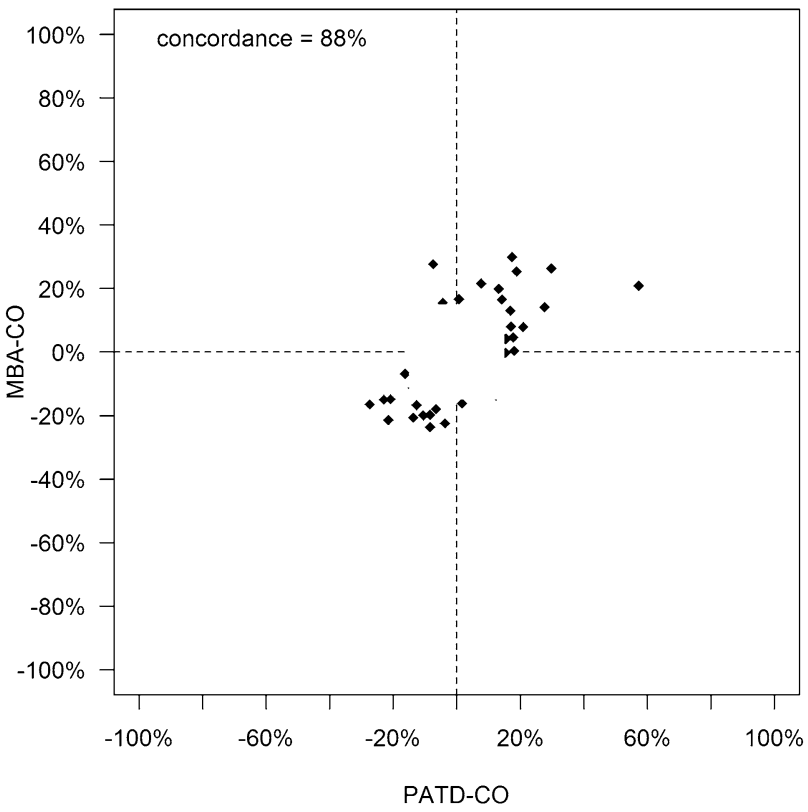

Fig. 3 The capability of the multi-beat analysis method to track relative changes in cardiac output is shown in a four-quadrant plot with a central exclusion zone of $15 \%$. MBA-CO, cardiac output estimations obtained with multi-beat analysis (test method); PATD-CO, cardiac output measurements with pulmonary artery thermodilution (reference method)

\section{Discussion}

In this method comparison study, $\mathrm{CO}$ estimations by multi-beat analysis of the radial arterial blood pressure waveform (Argos monitor) showed reasonable agreement and trending ability compared with $\mathrm{CO}$ measured by intermittent PATD in adult patients treated in the ICU after OPCAB.

This is the first prospective method comparison study evaluating the $\mathrm{CO}$ measurement performance of the novel multi-beat analysis algorithm now included in the recently FDA-cleared Argos monitor in comparison with intermittent PATD — the clinical reference method—in patients treated in the ICU after cardiac surgery. Previous studies investigated former versions of the multi-beat analysis algorithm in comparison with experimental gold standard CO measurements (aortic flow probe) in animals [13] and retrospectively in comparison with thermodilution $\mathrm{CO}$ measurements in 15 [12] and 169 [15] patients from a freely accessible ICU database. These previous studies showed promising results with regard to the $\mathrm{CO}$ measurement performance of the multi-beat analysis method. However, in these previous studies, the agreement and trending ability of the multi-beat analysis method with the reference method were assessed using calibrated multi-beat analysis-derived $\mathrm{CO}$ estimates, i.e., the $\mathrm{CO}$ estimates were calibrated to the reference $\mathrm{CO}$ values and multi-beat analysis was used as a calibrated pulse wave analysis method $[8,9]$. In contrast, in our study, we used multi-beat analysis as a-so-called-"uncalibrated" pulse wave analysis method only using arterial blood pressure waveform data and biometric information to estimate $\mathrm{CO}[8,9]$. Therefore, our findings are not indiscriminately comparable to results of these previous studies.

In 2010, Peyton and Chong [22] published an extensive meta-analysis on minimally-invasive methods for $\mathrm{CO}$ estimation in surgical and ICU patients. For pulse wave analysis methods, this meta-analysis yielded an overall pooled mean of the differences of $0.00 \mathrm{~L} / \mathrm{min}( \pm 95 \%$ confidence interval, $\pm 0.09 \mathrm{~L} / \mathrm{min}$ ), a pooled standard deviation of the differences between paired measurements of $1.22 \mathrm{~L} / \mathrm{min}$, and a pooled percentage error of $41.3 \%$ ( $\pm 95 \%$ confidence inter$\mathrm{val}, \pm 2.7 \%)$. Of note is that this meta-analysis included both uncalibrated and calibrated pulse wave analysis methods. Compared with these pooled results our study investigating uncalibrated multi-beat analysis revealed a slightly lower standard deviation of the mean of the differences and percentage error.

Four-quadrant plot analysis and the concordance rate are used to describe the ability of a test method to adequately and timely detect changes in a hemodynamic variable measured with a reference method. In our study, the concordance rate between changes in MBA-CO and PATD-CO $(88 \%)$ reflects good trending ability of multibeat analysis. The concordance rate is markedly higher than the ones observed in recent studies comparing the latest version of an established pulse wave analysis algorithm (FloTrac; Edwards Lifesciences, Irvine; CA, USA) 
with pulmonary artery thermodilution in patients having cardiac surgery using cardiopulmonary bypass [23, 24].

In general, irrespective of the specific method used, a key prerequisite for pulse wave analysis to provide accurate and reliable estimations of hemodynamic variables is an optimal signal quality of the arterial blood pressure waveform. Artifacts in the arterial blood pressure waveform can be caused by movement or by inappropriate damping properties (underdamping/resonance artifacts or overdamping) of the monitoring system consisting of the arterial catheter, a fluid-filled tubing system, and a pressure transducer [16, 25-27]. In accordance with our study, it has been demonstrated that underdamping/resonance artifacts occur in about one-third of cardiovascular patients in the operating room or ICU [26, 27]. Underdamping/resonance artifacts can result in a marked overestimation of systolic arterial blood pressure and incorrect estimation of pulse wave analysis-derived hemodynamic variables such as stroke volume, $\mathrm{CO}$, and pulse pressure variation [26, 27]. Therefore, during routine clinical care, we repeatedly perform "fast-flush tests" $[16,25]$ to evaluate the dynamic response of the arterial blood pressure monitoring system. To be able to objectively describe the actual measurement performance of a pulse wave analysis method in comparison with a reference method in a method comparison study it is essential to only include impeccable arterial blood pressure waveforms not disturbed by artifacts or inappropriate damping properties of the arterial catheter/tubing system. We, therefore, carefully checked the quality of the recorded arterial blood pressure waveforms and excluded waveform segments that were likely to be altered by inappropriate damping properties (underdamping/resonance or overdamping) and other artifacts. On the one hand, excluding artifactual waveforms is a key prerequisite to draw meaningful conclusions from method comparison studies on pulse wave analysis algorithms. On the other hand, excluding waveforms might increase the risk for selection bias and limit the generalizability of our findings to routine clinical conditions.

Another limitation of our study is that we did not use the multi-beat analysis monitor at the bedside, but recorded arterial waveform segments during routine care and later performed off-line analysis to estimate MBA-CO. In addition, because this was a single center study including patients after OPCAB surgery our results might not be generalizable to other settings or patient collectives. Further, our study setting was not designed to evaluate the trending ability of multi-beat analysis because the patients were in a relatively stable hemodynamic state and no CO-changing interventions were performed between study measurements. This resulted in a limited number of eligible observations for tending analysis.

\section{Conclusion}

CO estimations by multi-beat analysis of the radial arterial blood pressure waveform (Argos monitor) show reasonable agreement compared with $\mathrm{CO}$ measured by intermittent PATD in adult patients treated in the ICU after off-pump coronary artery bypass surgery (OPCAB). For further assessment of the trending ability additional studies are needed.

Acknowledgements We thank Ricardo Toledo-Crow, $\mathrm{PhD}$ (Advanced Science Research Center, City University of New York; New York, NY, USA) for his support with off-line analysis, i.e., feeding arterial blood pressure waveforms into the Argos CO monitor (Retia Medical) for $\mathrm{CO}$ estimation.

Funding The study was funded with an institutional restricted research grant by Retia Medical (Valhalla, NY, USA).

\section{Compliance with ethical standards}

Conflict of interest GG has received travel expenses from Vygon (Ecouen, France). SR has received honoraria for giving lectures from Baxter (Rome, Italy), BBraun (Melsungen, Germany), Orion Pharma (Espoo, Finland), Medigas (Assago, Italy), and Vygon. SR has received honoraria for consulting from Vygon and Medtronic (Minneapolis, MN, USA). SR has received institutional restricted research grants from Pall International (Milano, Italy) and Baxter (Rome, Italy). BS has received institutional restricted research grants from Retia Medical (Valhalla, NY, USA). BS collaborates with Pulsion Medical Systems (Feldkirchen, Germany) as a member of the medical advisory board and has received institutional restricted research grants, honoraria for giving lectures, and refunds of travel expenses from Pulsion Medical Systems. BS has received research support and honoraria for giving lectures from Edwards Lifesciences (Irvine, CA, USA). BS has received institutional restricted research grants, honoraria for giving lectures, and refunds of travel expenses from CNSystems Medizintechnik (Graz, Austria). BS has received institutional restricted research grants, honoraria for consulting, and refunds of travel expenses from Tensys Medical (San Diego, CA, USA). BS has received honoraria for giving lectures from Philips Medizin Systeme Böblingen (Böblingen, Germany). For all other authors there is no conflict of interest to declare.

Research involving human participants and/or animals This method comparison study was reviewed and approved by the ethics committee (Ethikkomission der Ärztekammer Hamburg, Hamburg, Germany). All procedures performed in studies involving human participants were in accordance with the ethical standards of the institutional and/or national research committee and with the 1964 Helsinki declaration and its later amendments or comparable ethical standards.

Informed consent Informed consent was obtained from all individual participants included in the study.

\section{References}

1. Cecconi M, De Backer D, Antonelli M, Beale R, Bakker J, Hofer C, Jaeschke R, Mebazaa A, Pinsky MR, Teboul JL, Vincent JL, 
Rhodes A. Consensus on circulatory shock and hemodynamic monitoring. Task force of the European Society of Intensive Care Medicine. Intensive Care Med. 2014;40(12):1795-815. https:// doi.org/10.1007/s00134-014-3525-z.

2. Gidwani UK, Mohanty B, Chatterjee K. The pulmonary artery catheter: a critical reappraisal. Cardiol Clin. 2013;31(4):545-65, viii. https://doi.org/10.1016/j.ccl.2013.07.008.

3. Reuter DA, Huang C, Edrich T, Shernan SK, Eltzschig HK. Cardiac output monitoring using indicator-dilution techniques: basics, limits, and perspectives. Anesth Analg. 2010;110(3):799-811. https://doi.org/10.1213/ANE.0b013e3181cc885a.

4. Rajaram SS, Desai NK, Kalra A, Gajera M, Cavanaugh SK, Brampton W, Young D, Harvey S, Rowan K. Pulmonary artery catheters for adult patients in intensive care. Cochrane Database Syst Rev. 2013;2:CD003408. https://doi.org/10.1002/14651858. cd003408.pub3.

5. Teboul JL, Saugel B, Cecconi M, De Backer D, Hofer CK, Monnet X, Perel A, Pinsky MR, Reuter DA, Rhodes A, Squara P, Vincent JL, Scheeren TW. Less invasive hemodynamic monitoring in critically ill patients. Intensive Care Med. 2016;42(9):1350-9. https:// doi.org/10.1007/s00134-016-4375-7.

6. De Backer D, Bakker J, Cecconi M, Hajjar L, Liu DW, Lobo S, Monnet X, Morelli A, Myatra SN, Perel A, Pinsky MR, Saugel B, Teboul JL, Vieillard-Baron A, Vincent JL. Alternatives to the Swan-Ganz catheter. Intensive Care Med. 2018;44:730-41. https ://doi.org/10.1007/s00134-018-5187-8.

7. Saugel B, Vincent JL. Cardiac output monitoring: how to choose the optimal method for the individual patient. Curr Opin Crit Care. 2018;24(3):165-72. https://doi.org/10.1097/mcc.00000 00000000492.

8. Thiele RH, Durieux ME. Arterial waveform analysis for the anesthesiologist: past, present, and future concepts. Anesth Analg. 2011;113(4):766-76. https://doi.org/10.1213/ANE.0b013e3182 2773ec.

9. Esper SA, Pinsky MR. Arterial waveform analysis. Best Practice Res Clin Anaesthesiol. 2014;28(4):363-80. https://doi. org/10.1016/j.bpa.2014.08.002.

10. Monnet X, Teboul JL. Passive leg raising: five rules, not a drop of fluid! Crit Care. 2015;19:18. https://doi.org/10.1186/s1305 4-014-0708-5.

11. Cecconi M, Parsons AK, Rhodes A. What is a fluid challenge? Curr Opin Crit Care. 2011;17(3):290-5. https://doi.org/10.1097/ MCC.0b013e32834699cd.

12. Lu Z, Mukkamala R. Continuous cardiac output monitoring in humans by invasive and noninvasive peripheral blood pressure waveform analysis. J Appl Physiol. 2006;101(2):598-608. https ://doi.org/10.1152/japplphysiol.01488.2005.

13. Mukkamala R, Reisner AT, Hojman HM, Mark RG, Cohen RJ. Continuous cardiac output monitoring by peripheral blood pressure waveform analysis. IEEE Trans Biomed Eng. 2006;53(3):459-67.

14. Mukkamala R, Kuiper J, Ahmad S, Lu Z. Cardiac output monitoring in intensive care patients by radial artery pressure waveform analysis. In: Proceedings of the 26th annual international conference of the IEEE. 2004. vol. 5, pp. 3712-15. https://doi. org/10.1109/iembs.2004.1404042.

15. Zhang G, Mukkamala R. Continuous and minimally invasive cardiac output monitoring by long time interval analysis of a radial arterial pressure waveform: assessment using a large, public intensive care unit patient database. Br J Anaesth. 2012;109(3):339-44. https://doi.org/10.1093/bja/aes099.

16. Gardner RM. Direct blood pressure measurement-dynamic response requirements. Anesthesiology. 1981;54(3):227-36.

17. Bland JM, Altman DG. Calculating correlation coefficients with repeated observations: part 1 -correlation within subjects. BMJ. 1995;310(6977):446.

18. Bland JM, Altman DG. Agreement between methods of measurement with multiple observations per individual. J Biopharm Stat. 2007;17(4):571-82. https://doi.org/10.1080/10543400701329422.

19. Critchley LA, Critchley JA. A meta-analysis of studies using bias and precision statistics to compare cardiac output measurement techniques. J Clin Monit Comput. 1999;15(2):85-91.

20. Saugel B, Grothe O, Wagner JY. Tracking changes in cardiac output: statistical considerations on the 4-quadrant plot and the polar plot methodology. Anesth Analg. 2015;121(2):514-24. https://doi. org/10.1213/ane.0000000000000725.

21. Montenij LJ, Buhre WF, Jansen JR, Kruitwagen CL, de Waal EE. Methodology of method comparison studies evaluating the validity of cardiac output monitors: a stepwise approach and checklist. Br J Anaesth. 2016;116(6):750-8. https://doi.org/10.1093/bja/ aew094.

22. Peyton PJ, Chong SW. Minimally invasive measurement of cardiac output during surgery and critical care: a meta-analysis of accuracy and precision. Anesthesiology. 2010;113(5):1220-35. https ://doi.org/10.1097/ALN.0b013e3181ee3130.

23. Lin SY, Chou AH, Tsai YF, Chang SW, Yang MW, Ting PC, Chen CY. Evaluation of the use of the fourth version FloTrac system in cardiac output measurement before and after cardiopulmonary bypass. J Clin Monit Comput. 2018;32(5):807-15. https://doi. org/10.1007/s10877-017-0071-6.

24. Kusaka Y, Ohchi F, Minami T. Evaluation of the fourth-generation FloTrac/Vigileo system in comparison with the intermittent bolus thermodilution method in patients undergoing cardiac surgery. J Cardiothorac Vasc Anesth. 2019;33(4):953-60. https://doi. org/10.1053/j.jvca.2018.06.017.

25. Ortega R, Connor C, Kotova F, Deng W, Lacerra C. Use of pressure transducers. N Eng J Med. 2017;376(14):e26.

26. Romagnoli S, Ricci Z, Quattrone D, Tofani L, Tujjar O, Villa G, Romano SM, De Gaudio AR. Accuracy of invasive arterial pressure monitoring in cardiovascular patients: an observational study. Crit Care. 2014;18(6):644.

27. Romagnoli S, Romano SM, Bevilacqua S, Lazzeri C, Gensini GF, Pratesi C, Quattrone D, Dini D, De Gaudio AR. Dynamic response of liquid-filled catheter systems for measurement of blood pressure: precision of measurements and reliability of the Pressure Recording Analytical Method with different disposable systems. J Crit Care. 2011;26(4):415-22.

Publisher's Note Springer Nature remains neutral with regard to jurisdictional claims in published maps and institutional affiliations. 